\title{
Qualitative Hybrid Control of Dynamic Bipedal Walking
}

\author{
Subramanian Ramamoorthy \\ Department of Electrical and Computer Engineering \\ The University of Texas at Austin \\ Austin, Texas 78712 \\ Email: s.ramamoorthy@mail.utexas.edu
}

\author{
Benjamin J. Kuipers \\ Department of Computer Sciences \\ The University of Texas at Austin \\ Austin, Texas 78712 \\ Email: kuipers@cs.utexas.edu
}

\begin{abstract}
We present a qualitative approach to the dynamical control of bipedal walking that allows us to combine the benefits of passive dynamic walkers with the ability to walk on uneven terrain. We demonstrate an online control strategy, synthesizing a stable walking gait along a sequence of irregularly spaced stepping stones. The passive dynamic walking paradigm has begun to establish itself as a useful approach to gait synthesis. Recently, researchers have begun to explore the problem of actuating these passive walkers, to extend their domain of applicability. The problem of applying this approach to applications involving uneven terrain remains unsolved and forms the focus of this paper. We demonstrate that through the use of qualitative descriptions of the task, the use of the nonlinear dynamics of the robot mechanism and a multiple model control strategy, it is possible to design gaits that can safely operate under realistic terrain conditions.
\end{abstract}

\section{INTRODUCTION}

A majority of land animals travel on legs [1] and they do so in a very efficient, reliable and graceful manner, with minimal cognitive effort and attention. Inspired by the success of legged animals, roboticists have long attempted to construct legged machines that are capable of operating in difficult environments, ranging from swamps to rescue sites. Over the years, several legged robots, often bipeds, have been constructed - to walk [2], hop [3], run [4] and even perform gymnastic maneuvers [5].

Bipedal walking robots are nonlinear underactuated dynamical systems. As described in [6], even simple walking machines are capable of surprising complexity. How does one compel these machines to behave as desired?

Traditionally, the problem of robotic walking has been solved in two stages. First, the nonlinear and nonholonomic aspects of the dynamics are actively compensated, e.g., via feedback linearization [7]. This yields a simpler linear system for which there exist several mathematically sophisticated control design techniques. This linear system also forms the basis for planning a trajectory to be tracked by the robot. Several techniques exist for this planning process, ranging from numerical optimization of parametric curves, e.g., BSplines, to temporal planning algorithms that operate on other discrete representations.

Notwithstanding the successes of this approach to gait design [8], there are some crucial limitations. Due to the need for continuously active control to compensate and eliminate the undesirable aspects of the dynamics, the resulting robot gaits are not very energy efficient. It has been observed [9] that such actively controlled robots utilize more than 10 times the power required by comparable biological walkers. Also, the process of implementing tracking controllers by compensating the natural dynamics requires high bandwidth and attention from the control computers, which places corresponding limits on the simplicity of the robots.

Biological walkers avoid these penalties of energy consumption and computational complexity by structuring the gait in a very different way - by skillfully utilizing, instead of overcoming, the nonlinearities in the physical mechanism.

Dynamic walking represents a promising alternate paradigm to gait synthesis that is based on utilizing the natural dynamics of the robot to reduce the complexity and increase the efficiency of gaits. Broadly speaking, dynamic walking is an approach to encoding the task in a language that is natural to the physical system [10] - the language of dynamical systems. In this setting, planning amounts to the selection of desirable trajectories in the natural dynamics of the system and active control is only required, occasionally, to correct deviations from these trajectories. In the passive dynamic walking literature, [9], [11], [12], [13], this encoding is often done empirically and largely through clever mechanical design possibly augmented by machine learning. This has already yielded interesting and useful results. However several open questions remain [13] - including that of synthesizing gaits that can handle irregular terrain such as might be encountered in rescue, planetary exploration and other applications.

In this paper, we present a novel gait synthesis strategy for dynamic bipedal walking. Through the use of natural nonlinear dynamics of the bipedal mechanisms, the analysis of these natural dynamics to extract control strategy primitives and the synthesis of composite control strategies, it is possible to design gaits that can safely operate under realistic terrain conditions. We believe this to be the first demonstration of intermittently actuated passive dynamic walking involving planned footfalls on irregular terrain. We demonstrate this on the task of stably walking on a sequence of irregularly spaced stepping stones. 


\section{SOME OBSERVATIONS REGARDING BIOLOGICAL AND BIOMIMETIC ROBOTIC WALKING}

Depending on one's perspective, bipedal locomotion may seem surprisingly difficult or trivially easy. Locomotion results from complex dynamical interactions between the walker and its environment. The walker, whether natural or artificial, is a complex multi-link mechanism. The environment is also complex and constantly changing. Yet, legged animals do not seem to be bogged down by this complexity.

Nature penetrates the wall of complexity through the clever use of synergies and symmetries. In [14], Full and Koditschek present a strong argument that animals handle complexity by devising control strategies based on dynamically simple template models and then embedding them into the higher dimensional physical system, the anchor. There is reason to believe that this is a generally applicable law of nature. Observations of infants [15] during their first walking months demonstrate that the process of learning to walk includes, as a critical step, the process of learning to reliably synergize their muscles to make each leg behave like a rigid pendulum.

Engineers would benefit greatly from adopting this paradigm. From the perspective of the designer of a robot, the goal of such a decomposition is to break down a complex control problem into two factored problems - (a) to design a safe adaptive strategy for a template model, the simplest model that is sufficiently expressive to capture all necessary design specifications while also being dynamically equivalent to the anchor model, and (b) to embed this strategy in the higher dimensional anchor model. This paper focuses on the first problem and aims to present a general and extensible solution. Once the first problem is solved, several possible strategies may be adopted to implement the strategy in more complex systems. A very appealing approach, presented in [2], is to define dynamical primitives that apply to simpler subsystems - the global dynamics being composed from such primitives. However, there is an unfulfilled need for a design methodology that could be used to reason about the global behavior of such strategies. This is crucial if we wish to handle realistic types of irregular terrain, and we address this issue.

The dominant hypothesis regarding templates for bipedal walking is that each leg acts as a pendulum, executing a "compass gait" [16], [17] - walking consists of a synchronized sequence of vaulting over an inverted pendulum and swinging a suspended pendulum. Roboticists have constructed systems that are direct physical embodiments of this model, e.g., [12]. In [9], we find mechanical embodiments of actuated versions of simple compass gait robots and an empirical demonstration that they are capable of walking on flat ground. While these machines are elegantly simple, they are also fragile and unsuitable for rugged environments. In [6], we find mathematical analysis of the dynamics of this class of robots, called simple walkers. The walker is analyzed as a nonlinear system, to locate periodic orbits whose stability is understood in terms of convergence in a Poincare map. This analysis provides insight but it does not make it possible for the walker to navigate rugged terrain. Nonetheless, the work on passive walking highlights an organizing principle in nature - the dynamics of the interaction with the environment can be gainfully utilized to implement energy storage and exchange mechanisms, bringing down total energy consumption [1]. Even in situations where energy storage mechanisms, e.g., springs, are not physically available in the robot, it is often more energy efficient to simulate the passive component than it is to pursue alternate active strategies.

Walking in the real world requires tradeoffs [1]. In animals, we see tradeoffs between energy efficiency and the need for rapid reliable maneuvers, e.g., while escaping from a predator. In robotics, we seek the ability to build machines that can perform useful tasks as they walk, e.g., bring a cup of coffee while simultaneously avoiding obstacles on the floor. This suggests that gaits ought to be designed to be capable of rapid adaptation to a changing environment. It has been challenging to implement such adaptation while maintaining safety. Robots that are designed according to the passive/simple walking paradigm and are amenable to principled analysis are not well suited to unstructured environments. Correspondingly, several empirically successful adaptive machines have no clear basis for stability or correctness arguments. We propose a way to merge the two paradigms.

\section{A QUALITATIVE HYBRID CONTROL STRATEGY FOR DYNAMIC BIPEDAL WALKING}

At the core of this problem is the task of generating, in a finite horizon setting, a sequence of control actions that induces safe walking over irregular terrain. This is a motion planning problem with several possible solutions.

A crucial assumption that is made in nearly all existing approaches to robotic walking is that walking results from a limit cycle behavior. In passive walkers [11], [12], this limit cycle is numerically computed and an appropriately initialized machine converges to this cycle. In actively controlled walkers [8], such a limit cycle is enforced by feedback compensation. However, on irregular terrain and especially in settings where planned footfalls need to be placed on a specific sequence of locations, it is not clear that there is a single limit cycle that should have a preferred position with all deviations being actively compensated [1]. Instead, we argue that it is beneficial to define a family of trajectory segments from which elements may be selected at the beginning of each step, in order to achieve specific goals. An exactly periodic limit cycle could certainly be composed in this way. However, such a hybrid strategy is also capable of several other trajectories, forming a richer family. The notion of composing hybrid control strategies using families of local controllers has been explored in [18], [19]. In this paper, we apply this concept in the intermittently actuated passive dynamics setting.

In this section, we will present an algorithm that implements this approach. Before doing that, we will present a model of a bipedal walker and make some observations regarding its dynamical behavior which motivates the structure of the algorithm. 


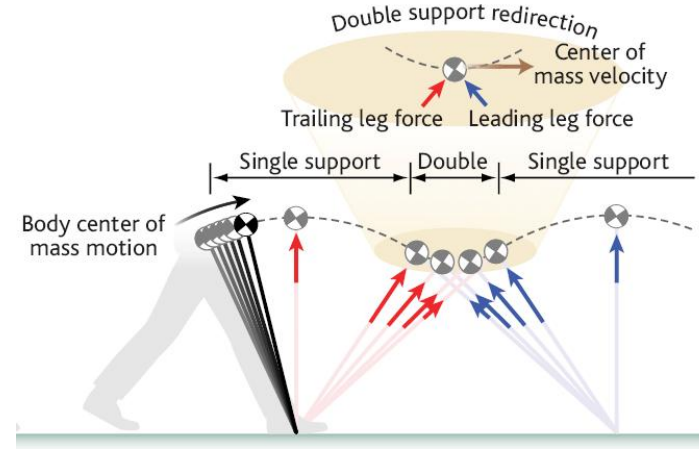

Fig. 1. Conceptual schematic of the compass gait model of human walking (Reproduced from Kuo, A.D., Science 309:1686-1687).

\section{A. Template model for bipedal walking}

Walking is a process of vaulting over an inverted pendulum while simultaneously swinging another pendulum, in a synchronized fashion. Various aspects of this pendulum like behavior have been investigated in the biomechanics literature [16], [17], [11], [20], [21]. These ideas have also received considerable attention within the robotics community [9], [6]. We use a slightly modified version of this model. Figure 1 illustrates the concept of the compass gait model.

Figure 2 depicts the corresponding pendulum system abstraction. It consists of three point masses, one corresponding to the torso and two corresponding to the legs and feet. In the swing phase, one foot is on the ground and the system has only two moving masses, the torso and the swinging foot. Viewed from the vantage point of the stationary foot, the system looks like a double pendulum (when not actuated) and like the acrobot (when actuated). However, there is one difference in that each leg/link is modeled as being capable of achieving any desired length quickly, to overcome the terrain. A retraction in this model would correspond to a movement of the foot and bending of the knee in a multi-link robot. In numerical experiments, we will use a noticeable retraction (leg length at $80 \%$ of full extension, i.e., $\rho=0.8$ ). However, this variable may be tuned to the terrain conditions.

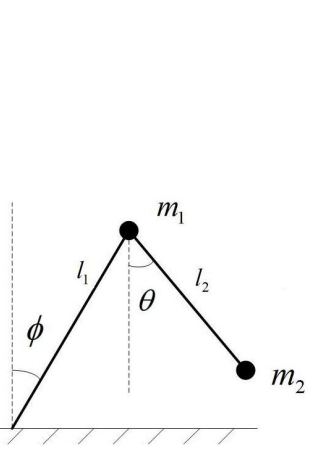

(a)

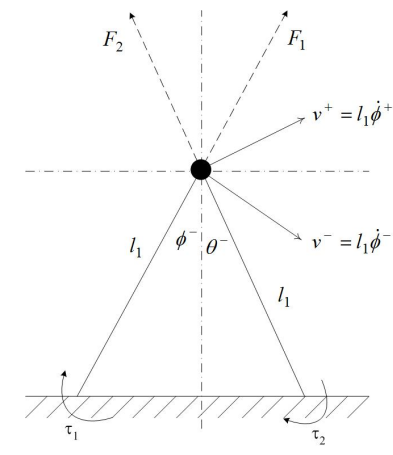

(b)
Fig. 2. Schematic of the dynamic model of the simple walking machine along with the relevant variables. Parts $a$ and $b$ correspond to the single support and double support phases, respectively.
For the single support phase, the equations of motion for this system can be derived using the Euler-Lagrange equations that encode the physical principle of least action [22]. The Lagrangian is the difference between kinetic and potential energies of the system,

$$
\begin{array}{r}
L=\frac{1}{2}\left(m_{1}+m_{2}\right) l_{1}{ }^{2} \dot{\phi}^{2}+\frac{1}{2} m_{2} l_{2}{ }^{2} \dot{\theta}^{2}+m_{2} l_{1} l_{2} \dot{\phi} \dot{\theta} \cos (\phi+\theta) \\
-\left(m_{1}+m_{2}\right) g l_{1} \cos \phi+m_{2} g l_{2} \cos \theta
\end{array}
$$

where $\theta$ is the angle made by the swing leg with the vertical axis and $\phi$ is the angle made by the stance leg with the vertical axis.

From $L$, we can derive the equations of motion as two coupled nonlinear ordinary differential equations. With, $\mu=$ $\frac{m_{2}}{m_{1}+m_{2}}, \rho=\frac{l_{2}}{l_{1}}, k_{1}=\frac{g}{l_{1}}, \alpha_{1}=\frac{1}{\left(m_{1}+m_{2}\right) l_{1}{ }^{2}}$ and $\alpha_{2}=\frac{1}{m_{2} l_{1}{ }^{2}}$, we express the equations of motion as,

$$
\begin{array}{r}
\ddot{\phi}-k_{1} \sin \phi=\Delta_{1} \\
\ddot{\theta}+\frac{k_{1}}{\rho} \sin \theta=\Delta_{2} \\
\Delta_{1}=-\mu \rho \cos (\phi+\theta) \ddot{\theta}+\mu \rho \sin (\phi+\theta) \dot{\theta}^{2}+\alpha_{1} \tau_{\phi} \\
\Delta_{2}=-\frac{1}{\rho} \cos (\phi+\theta) \ddot{\phi}+\frac{1}{\rho} \sin (\phi+\theta) \dot{\phi}^{2}+\frac{\alpha_{2}}{\rho^{2}} \tau_{\theta}
\end{array}
$$

where $\tau_{\theta}$ and $\tau_{\phi}$ are "hip" and "ankle" torques. The ankle is not actuated, i.e., $\tau_{\phi}=0$, for the purposes of handling terrain uncertainty, although it remains available for lower level controllers to compensate noise, etc.

These equations describe two pendulum subsystems, one inverted and one suspended, each perturbed by the other. In most realistic walking systems, $\mu \rho \sim 0$, so that $\Delta_{1} \sim 0$ when $\tau_{\phi}=0$. However, the effect of the stance leg on the swing leg, $\Delta_{2}$, need not be negligible. In fact, this coupling term continues to influence the motion once the system is in single support.

In the double support phase, both legs are on the ground and the system dynamics is much more constrained. For our purposes, the most relevant dynamics is that of the torso, which has four forces and torques acting on it to redirect its motion. The system comes into double support with a state, $\left(\phi^{-}, \theta^{-}, \dot{\phi}^{-}, \dot{\theta}^{-}\right)$. Following [21] and [23], the double support phase is treated as being short enough that the configuration of legs is roughly unchanged (hence, after swapping roles, $\phi^{+}=$ $-\theta^{-}, \theta^{+}=-\phi^{-}$) but long enough that feasibly bounded forces can be applied to influence the velocity variables.

The primary mode of actuation in our strategy is to apply $F_{1}, F_{2}, \tau_{1}, \tau_{2}$ during double support, which takes a nonzero time, $\delta t_{D S P}$. These quantities may be computed to achieve a desired $\dot{\phi}^{+}$. Equating the applied forces to the rate of change of momentum during double support, and rearranging terms, 


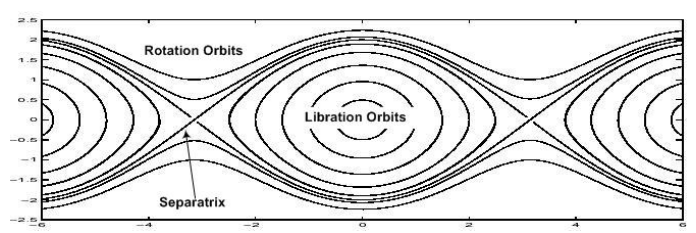

Fig. 3. Phase portrait $(\theta-\dot{\theta})$ of the frictionless simple pendulum.

we get,

$$
\begin{array}{r}
\left(\begin{array}{cccc}
\cos \phi^{-} & \cos \theta^{-} & \frac{-\sin \phi^{-}}{l_{1}} & \frac{-\sin \theta^{-}}{l_{1}} \\
\sin \phi^{-} & -\sin \theta^{-} & \frac{-\cos \phi^{-}}{l_{1}} & \frac{-\cos \theta^{-}}{l_{1}}
\end{array}\right)\left(\begin{array}{c}
F_{1} \\
F_{2} \\
\tau_{1} \\
\tau_{2}
\end{array}\right) \\
= \\
\frac{m_{1} l_{1}}{\delta t_{D S P}}\left(\begin{array}{cc}
\sin \theta^{-} & \sin \phi^{-} \\
\cos \theta^{-} & -\cos \phi^{-}
\end{array}\right)\left(\begin{array}{c}
\dot{\phi}^{+} \\
\dot{\phi}^{-}
\end{array}\right)+\left(\begin{array}{c}
m_{1} g \\
0
\end{array}\right)
\end{array}
$$

A similar analysis yields the torque $\tau_{\theta}$ to achieve desired $\dot{\theta}^{+}$. With the exception of these short pulses during double support, the system dynamics will be permitted to evolve naturally without actuation. This is consistent with empirical observations of human walking. In biological experiments [24], [20], [21] it has been observed that muscles are silent during most of the swing phase and all activity is restricted to short intervals at the beginning and end of the swing. Similarly, the use of toe-off forces, biologically implemented via plantarflexion, as the primary means of actuating stance leg velocity is also consistent with biological theories of walking.

The equations of motion define a nonlinear map, in terms of the state space, $\mathcal{S}_{1} \equiv\{\phi, \theta, \dot{\phi}, \dot{\theta}\}$, a subspace, $\mathcal{S}_{2}=$ $\{\dot{\phi}, \dot{\theta}\}$ and the action space, $\mathcal{A}=\left\{F_{1}, F_{2}, \tau_{1}, \tau_{2}, \tau_{\theta}\right\}$. The dynamic evolution of the system yields mappings between these spaces. In particular, the single support phase represents the uncontrolled map, $\mathcal{M}_{\mathcal{S S P}}: \mathcal{S}_{1} \mapsto \mathcal{S}_{1}$ and the double support phase represents a map, $\mathcal{M}_{\mathcal{D S P}}: \mathcal{S}_{2} \times \mathcal{A} \mapsto \mathcal{S}_{2}$ which does not influence the kinematic variables, $\theta$ and $\phi$. At the algorithmic level and for planning purposes, it is assumed that these maps admit functional evaluation, either through a dynamic simulation or by experiments on a physical robot.

\section{B. Dynamic behaviors of the template model}

The pendulum is a simple Hamiltonian system and its phase space behavior can be completely understood in terms of two families of orbits, called the libration and rotation orbits, separated by a single trajectory called the separatrix (the singular homoclinic orbit that connects the hyperbolic, i.e., $-\pi$ and $\pi$, fixed points). Figure 3 depicts the phase space of the frictionless simple pendulum. All closed orbits "inside" the separatrix belong to the family of libration orbits and all other periodic orbits, "outside" the separatrix, belong to the family of rotation orbits. Each of these orbits is uniquely specified by the energy of the system (which corresponds to initial position and velocity of the pendulum).

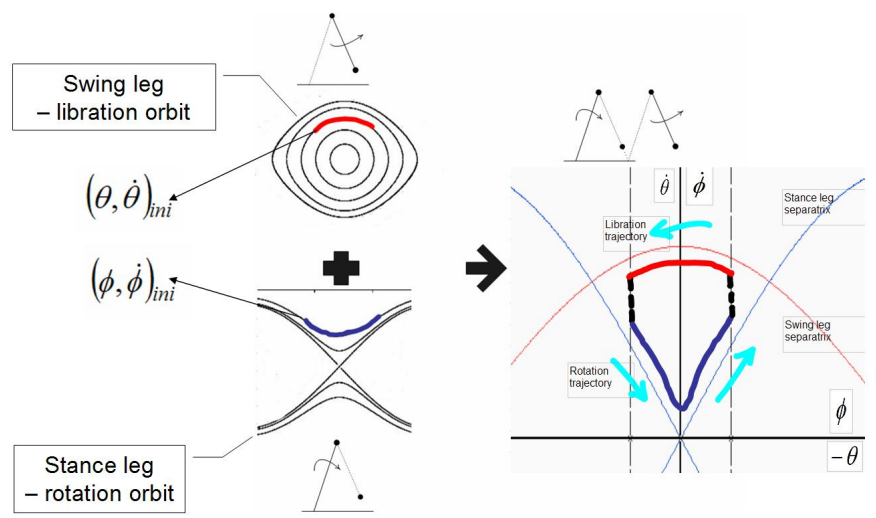

Fig. 4. Synthesizing composite trajectories based on selections from families of qualitatively similar orbits. This figure depicts only a few representative rotation and libration trajectories. In fact, these are dense families and there are infinitely many such curves to choose from. Note that, for clarity of exposition, the trajectory segments have been aligned and transition is a simple vertical line. In general, these trajectories could be asymmetrical and aligned differently along the velocity axis, depending on kinematic conditions and terrain.

The behavior of the stance leg of the compass gait biped is represented by the family of rotation orbits, and the swing leg behavior is represented by libration orbits. A composite orbit obtained by switching between representative trajectories from these qualitatively defined families of orbits is capable of describing the walking behavior. Of course, not all compositions will be admissible and further conditions will be necessary to achieve foot placement goals.

This process of composition is depicted in figure 4. The swing leg and stance leg trajectories will have to synchronize for the robot to successfully walk. Further constraints, e.g., desired step length, will enter this picture indirectly, as constraints on the sets from which trajectory segments may be chosen. This composition results in mappings of the form, $\mathcal{M}_{\mathcal{D S P}} \circ \mathcal{M}_{\mathcal{S S P}}: \mathcal{S}_{1} \times \mathcal{A} \mapsto \mathcal{S}_{1}$. Steady walking consists of a possibly infinite composition, $\mathcal{M}_{\mathcal{D S P}} \circ \ldots \circ \mathcal{M}_{\mathcal{S S P}} \circ \mathcal{M}_{\mathcal{D S P}}$ : $\mathcal{S}_{1} \times \mathcal{A} \mapsto \mathcal{S}_{1}$. The goal of planning is to select a policy of forces and torques that will impart certain properties to this map.

The basic property we wish to ensure in these maps is that the swing leg and stance leg trajectory segments are synchronized, i.e., they take the same amount of time to reach the planned footfall position. For a pendulum, the swing time may be determined analytically. In terms of a parameter describing energy, $\kappa=\sqrt{\frac{1}{2}\left(1+\frac{E}{m g l}\right)}$ (where $m$ and $l$ stand for $m_{1} / m_{2}$ and $l_{1} / l_{2}$, as appropriate), we have for small angles,

$$
\tau(\kappa)= \begin{cases}2 \pi \sqrt{\frac{l}{g}} & \kappa<1 \\ \frac{\pi}{\kappa} \sqrt{\frac{l}{g}} & \kappa>1\end{cases}
$$


and for larger angles,

$$
T(\kappa, \tau)=\left\{\begin{array}{cc}
\tau \frac{K(\kappa)}{K(0)} & \kappa<1 \\
\tau \frac{K\left(\kappa^{-1}\right)}{K(0)} & \kappa>1
\end{array}\right.
$$

where $K(\kappa)=\int_{0}^{\frac{\pi}{2}} \frac{d \theta}{\sqrt{1-\kappa^{2} \sin \theta^{2}}}$.

We could achieve synchronization of the swing and stance legs by setting the energy appropriately. Given analytical expressions of this form, computing the forces and torques is simply a matter of equating two such quantities and solving for the unknowns.

However, this argument assumes that the stance and swing legs are decoupled. In fact, the swing leg is driven at the pivot by the movement of the torso. This complicates the dynamics of the swing leg and poses problems for a passive dynamics framework that avoids actuating the swing leg, except to set an initial velocity as it enters single support.

It is well known that the driven pendulum is chaotic [22], implying the possibility that the families of trajectories we deal with may be more complex than indicated in figure 4 . In the worst case, this could invalidate our hypothesis about the possibility of synthesizing composite gaits from qualitatively defined families of orbits.

The source of this chaos is a resonance between the natural periodic dynamics and the periodic components of the external driving force. A key feature of the transition to chaos [25] is that it is gradual. As the driving amplitude is smoothly varied from a small value, only a few trajectories near critical points and the separatrix are affected. Chaos begins here and slowly affects the rest of phase space. For our purposes, this implies that families of topologically equivalent orbits do not suddenly disappear. Instead, these regions gradually shrink. Eventually, as the driving amplitude increases, no meaningful qualitative statements can be made - global unpredictability sets in. If we can identify operating regimes and phase space regions where regular trajectories persist then we can still implement the strategy discussed so far, safely ignoring the more complex dynamics in other regions of phase space.

It is possible to estimate the regions of phase space where such complex dynamics exists, and avoid it. One approach is to estimate the width of the separatrix layer [25] and suitably restrict $\dot{\theta}$ to avoid this region. Another approach is to reason about the nature of the individual solutions. For instance, it is a theorem [26] that, if $f(t)$ is a $\tau$-periodic function and $\ddot{v}(t)+\sin v(t)=0$ admits a $j \tau$ periodic solution for some positive integer $j$, then for sufficiently small $|\epsilon|$, $\ddot{u}(t)+\sin u(t)=\epsilon f(t)$ admits at least two solutions that are slightly shifted versions of the original solution, $Z=$ $\{v(\bullet+\psi): \psi \in \Re\}$. Through the use of such ideas, it is possible to establish bounds on $\dot{\theta}, \dot{\phi}$ so that the distortion of the family of libration orbits may be bounded, providing a continuous selection of swing leg trajectories. In a companion paper, we will make this argument rigorously with relevant proofs. For the current purposes, we provide a visual depiction of the above statement using a simple numerical experiment.
Our method is to observe the phase space behaviors through the evolution of randomly distributed points in phase space. By allowing an ensemble of points to evolve for a short period of time, we are able to visualize the deformation of the phase portrait. Figure 5 depicts a representative result of these experiments. We have overlaid the original trajectory shapes from figure 4 over our random dots results to aid visualization.

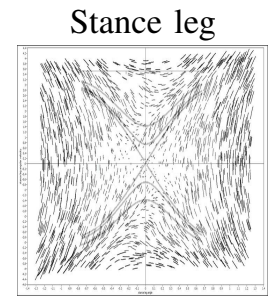

(a)

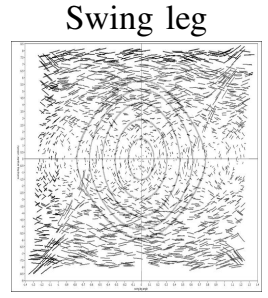

(b)

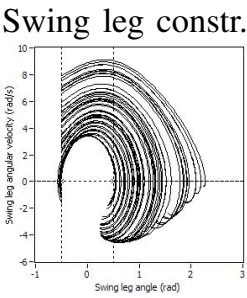

(c)
Fig. 5. Random dots based phase portraits for the nonlinear system of equations 2 and 3. Phase space trajectories representing the unperturbed pendulum have been overlaid on the results to enable visualization of the deformation due to chaos. Note that these phase portraits correspond to the full 4D system. Each plot represents a projection onto a 2D phase plane, with angular position as the x-axis and angular velocity as the y-axis. As seen in part $b$, the swing leg phase space has been significantly perturbed. However, by placing the constraints outlined in section III-B, we are able to extract a family of regular trajectories, as shown for the swing leg in part $c$.

Regular regimes correspond to bounded $\dot{\phi}, \dot{\theta}$, i.e., bounded subsets of $\mathcal{S}_{1}$. In particular, the following constraints will yield an operating region in state space where the original description of figure 4 will be essentially correct:

- Swing and stance leg angles, $\phi, \theta$, are restricted to a magnitude of $45^{\circ}$, from basic mechanical considerations.

- The stance leg trajectories are restricted to "separatrixlike" trajectories, i.e., to a manifold defined by $\dot{\phi}=$ $\beta \sqrt{\frac{2 g}{l_{1}}(1-\cos \phi)}$, where $\beta=1$ describes the separatrix. This equation defines the chosen value of $\dot{\phi}^{+}$in the double support phase.

- The magnitude of swing leg velocity, $\dot{\theta}$, is bounded above in our experiments $[0,8] \mathrm{rad} / \mathrm{s}$.

\section{An Online Adaptive Algorithm for Dynamic Walking}

We are now able to describe the motion planning algorithm. The concrete problem we solve is that of walking on a series of footholds, presented sequentially - one step at a time, in terms of a desired displacement $\left(x_{d}, y_{d}\right)$ measured from the current stance leg foothold. There are several ways to select footholds, based on a variety of considerations that are beyond the scope of this paper. However, footholds selected by any technique can be translated to a requirement in $\left(x_{d}, y_{d}\right)$ and so our algorithm can be applied.

Beginning from an initial state, $\phi^{-}, \theta^{-}, \dot{\phi}^{-}, \dot{\theta}^{-}$, with a particular assignment of swing and stance legs (e.g., stance $=$ right, swing $=$ left), this algorithm executes the following steps in a loop:

1) (Double Support) 
a) Apply $F_{1}, F_{2}, \tau_{1}, \tau_{2}$ to implement desired value of $\dot{\phi}^{+}$. The stance leg velocity is given by $\dot{\phi}^{+}=$ $\beta \sqrt{\frac{2 g}{l_{1}}\left(1-\cos \phi^{+}\right)}$. The desired swing leg velocity, $\dot{\theta}^{+}$, will be computed by numerical optimization, described below in more detail. This value can be achieved using $\tau_{\theta}$. This represents the execution of the map, $\mathcal{M}_{\mathcal{D S P}}: \mathcal{S}_{2} \times \mathcal{A} \mapsto \mathcal{S}_{2}$.

2) (Single Support)

a) Retract the swing leg to length $l_{2}$.

b) Allow the dynamics to evolve passively, according to the map $\mathcal{M}_{\mathcal{S S P}}: \mathcal{S}_{1} \mapsto \mathcal{S}_{1}$, until a stopping condition is reached (to be described below).

c) When this stopping condition is reached, assign new values to $\phi^{-}, \theta^{-}, \dot{\phi}^{-}, \dot{\theta}^{-}$and extend the swing leg from length $l_{2}$ to $l_{1}$.

3) (Transition)

a) Swap the roles of legs (e.g., for the first step, we will have stance $=$ left and swing $=$ right) and assign $\phi^{+}=-\theta^{-}, \theta^{+}=-\phi^{-}$.

The only step involving active control with energy injection/dissipation is $1 \mathrm{a}$, representing the application of constant forces and torques for a small period of time, $\delta t_{D S P}$. In our experiments, we fix this time period in order to compute forces and torques. However, this value is a variable that can be changed for other experiments, perhaps based on empirical data.

The angular velocity, $\dot{\theta}^{+}$, and the separatrix scaling factor, $\beta$, are determined by constrained optimization. A dynamic simulation is used to evaluate a cost function that encodes task specifications. In essence, optimization computes $\mathcal{M}_{\mathcal{S S P}}{ }^{-1}$. The dynamic simulation for $\mathcal{M}_{\mathcal{S S P}}$ is executed until a stopping condition is reached, specified by geometrical considerations. The requirement is that $\phi(t)=\phi_{\text {des }}$ and $\theta(t)=\theta_{\text {des }}$ for some value of $t$. If $A\left(x_{1}, y_{1}\right), B\left(x_{2}, y_{2}\right)$ and $\mathrm{C}\left(x_{d}, y_{d}\right)$ are the coordinates of the torso COM, swinging foot and desired foothold respectively, then $\phi_{\text {des }}$ and $\theta_{\text {des }}$ result from the consideration that $\mathrm{AB}$ and $\mathrm{BC}$ lie on the same line and $\mathrm{AC}$ has length $l_{1}$. We will use the former requirement and terminate the simulation when $x_{d}=x_{1}+\frac{\left(y_{d}-y_{1}\right)\left(x_{2}-x_{1}\right)}{\left(y_{2}-y_{1}\right)}$. The constraint that $l_{1}=|A C|$ is handled in the optimization.

If $\gamma=\left\{\dot{\theta}^{+}, \beta\right\}$, then the evolution of the dynamics determines the values of $\phi_{\gamma}(t)$ and $\theta_{\gamma}(t)$. If the single support phase operates during the time interval, $t \in\left[t_{1}, t_{2}\right]$, then we require that, $\gamma=\arg \min \mid w_{1}\left(x_{d}-\left[x_{3}\left(t_{2}\right)\right]_{\gamma}\right)^{2}+w_{2}\left(y_{d}-\right.$ $\left.\left[y_{3}\left(t_{2}\right)\right]_{\gamma}\right)^{2}+w_{3}\left(\dot{\phi}_{\gamma}^{+}\right)^{2} \mid,\left(x_{3}, y_{3}\right)$ being the coordinate of the point of footfall. This needs to be a constrained minimization. In addition to the constraints mentioned in section III-B, we need to account for finite actuator strengths and unilateral force constraints, i.e., $0<F_{1}<F_{\max }, 0<F_{2}<F_{\max },\left|\tau_{1}\right|<$ $\tau_{\max },\left|\tau_{2}\right|<\tau_{\max }$.

Given values of $\dot{\phi}^{-}, \dot{\phi}^{+}$, equation 6 sets up a force assignment problem. We solve this problem to obtain a minimum norm solution, by solving the quadratic program, min $x^{\prime} I x$ such that $A x=b$, where $x=\left\{F_{1}, F_{2}, \tau_{1}, \tau_{2}\right\}^{\prime}$ and $A, b$ result from collecting the terms in equation 6 .
The procedure adopted for the constrained optimization problem is as follows:

1) Generate $n$ uniformly distributed values for $\gamma=\left\{\dot{\theta}^{+}, \beta\right\}$ and evaluate $J=\mid w_{1}\left(x_{d}-\left[x_{3}\left(t_{2}\right)\right]_{\gamma}\right)^{2}+w_{2}\left(y_{d}-\right.$ $\left.\left[y_{3}\left(t_{2}\right)\right]_{\gamma}\right)^{2}+w_{3}\left(\dot{\phi}_{\gamma}^{+}\right)^{2}$

2) With $J_{1}=\left|w_{1}\left(x_{d}-\left[x_{3}\left(t_{2}\right)\right]_{\gamma}\right)^{2}+w_{2}\left(y_{d}-\left[y_{3}\left(t_{2}\right)\right]_{\gamma}\right)^{2}\right|$ and $J_{2}=\left|w_{3}\left(\dot{\phi}_{\gamma}^{+}\right)^{2}\right|$, compute the best multiobjective optimum from the above set (subject to constraints mentioned above) using a vector distance from the origin, $\sqrt{J_{1}^{2}+J_{2}^{2}}$.

3) Solve a sequential quadratic program using the $\gamma=$ $\left\{\dot{\theta}^{+}, \beta\right\}$ from the previous step to refine the solution.

Solving this problem amounts to achieving the kinematic specification $\left(x_{d}, y_{d}\right)$ through the use of a low energy, rotation trajectory for the stance leg. The kinematic specifications specify a curve in the 2-dimensional space of $\gamma$, not a unique point. An optimization problem that is based on just this requirement (with $w_{3}=0$ ) would be underspecified and the solution would oscillate along this feasible curve. This is undesirable because it implies significant unnecessary force expenditure to switch between orbits. The term, $w_{3}\left(\dot{\phi}^{+}\right)^{2}$, is intended to encourage convergence towards a low energy gait, in keeping with our goal of energy efficiency. In a multiobjective and multi-task environment, the above cost functions may be augmented without impacting the structure of the algorithm.

The reader may observe that the above procedure involves some computational complexity, which would seem to be at odds with our stated goal of simplicity. In this context, we remark that the above algorithm may be used offline to acquire a mapping between $\alpha=\left(x_{d}, y_{d}\right)$ and $\gamma=\left\{\dot{\theta}^{+}, \beta\right\}$, e.g., radial basis functions of the form $\gamma=\frac{\Sigma_{i} w_{i}(\alpha) \gamma_{i}}{\Sigma_{i} w_{i}(\alpha)}$ where $w_{i}(\alpha)=\frac{1}{\sqrt{2 \pi}} \exp \frac{-\left(\alpha-\alpha_{i}\right)^{2}}{\sigma^{2}}$. A map of this form is a fairly simple representation of the strategy that may be evaluated efficiently, allowing the above algorithm to be used online in realistic robots. In biology, such a map may be acquired developmentally, through continuous learning. The point of the design in this paper is to constructively arrive at a similar result, in such a way that we are able to reason about dynamical behavior.

\section{THE STEPPING STONES EXPERIMENT}

We evaluate this algorithm by simulating dynamic walking under progressively more irregular terrain conditions - going from flat ground, to climbing a set of regularly spaced stairs to walking on random terrain. In each of these simulations, the robot begins at rest. In every case, the maximum $x$-error is within $0.9 \mathrm{~mm}$ and the maximum $y$-error is within $0.3 \mathrm{~mm}$. These are tight error bounds (for a robot with $l_{1}=0.84 \mathrm{~m}$ ) and suggest that this algorithm can be safely used in situations where only small discontinuous footholds are available.

The base case experiment is to make the robot walk along equally spaced footholds on flat ground. For this case, figure 6 depicts the phase space behavior (corresponding to the conceptual schematic of figure 4). 


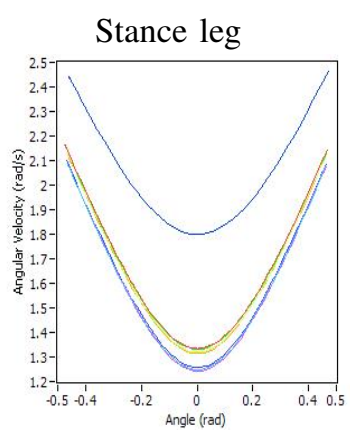

(a)

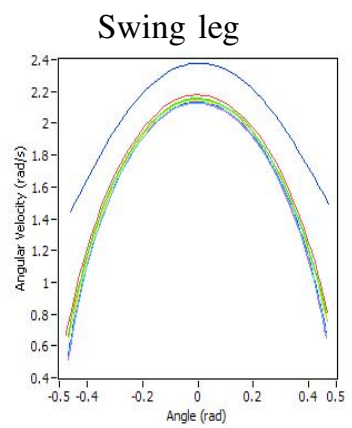

(b)
Fig. 6. Hybrid phase space trajectories for simple flat ground walking. Notice that, barring transients, the trajectories settle into a stable limit cycle comparable to that resulting from traditional techniques.

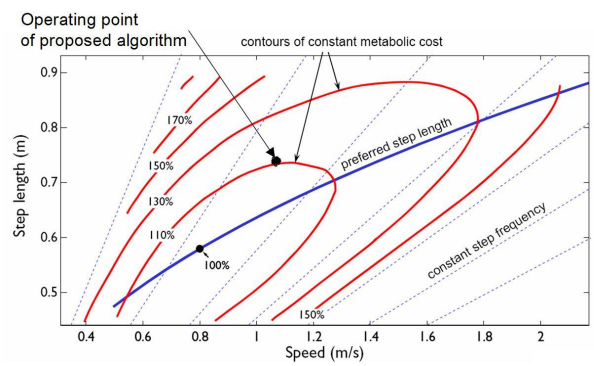

Fig. 7. Relationship between preferred forward speed and step length in human walking. The blue line depicts empirical data that also minimizes metabolic cost. The absolute minimum and the operating point of the proposed algorithm are depicted by black dots.

This corresponds to a forward velocity of approximately 1.1 $\mathrm{m} / \mathrm{s}$. It is known [21] that humans adopt a preferred speed for each step length. This relationship is depicted in figure 7. We remark that the speed chosen by the proposed algorithm is only slightly above the preferred speed curve for humans, at the $110 \%$ metabolic cost level. This implies that not only is the algorithm capable of recovering the stable limit cycle behavior of other walking control strategies, but also, it naturally results in realistic velocity behavior.

Next, we make the robot climb a flight of stairs, with results depicted in figures 8 and 9. From figure 7, we note the empirical observation that humans walk faster when step lengths increase. This is indeed our observation as well and the trajectories cluster around two different orbits according to this change in speed.

Lastly, figures 10 and 11 depict results for irregular footholds in two dimensions. Due to the wide variations in footholds, each step requires a different trajectory. We believe this to be the first demonstration of actuated passive walking on an irregular sequence of footholds.

The stability or correctness of gaits resulting from the proposed strategy may be characterized by the availability of a nonempty family of feasible trajectories, given a realization of the foothold sequence. Formally proving the existence of such a family for all (or for a set of well-defined) irregular

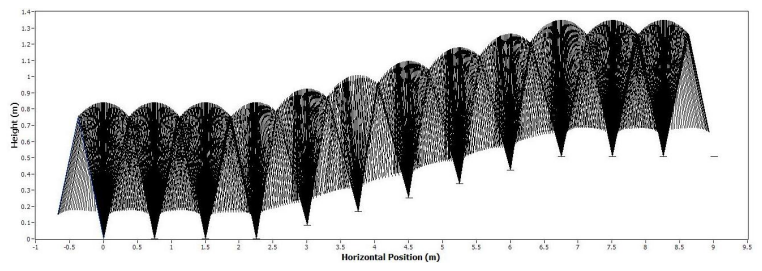

Fig. 8. Configuration, over time, of 12 steps of the simulated robot climbing a flight of stairs.

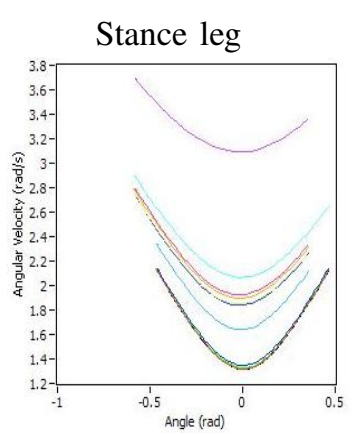

(a)

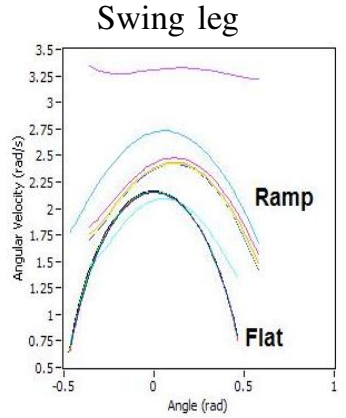

(b)
Fig. 9. Hybrid phase space trajectories for stair climbing. Notice that trajectories separate into two categories, for flat ground and ramp respectively.

terrain conditions is an open question. This is a part of our current investigations.

\section{DISCUSSION}

\section{A. Benefits of qualitative representation of dynamics}

In this work, we have depended substantially on the qualitative structure of the nonlinear dynamics. The idea that there exists a family of possible periodic orbits is certainly not new. The novel idea in our work, we believe, is that the desired orbits may be constructed, online, by composing multiple trajectory segments that admit qualitative descriptions. By identifying families of elemental trajectory segments, we have found a way to construct orbits, that may be periodic, quasiperiodic or even aperiodic, as required. The composite orbit accommodates changing task requirements. Yet, due to the existence of a dense family of orbits, we have not sacrificed the core stability properties that are essential for this task.

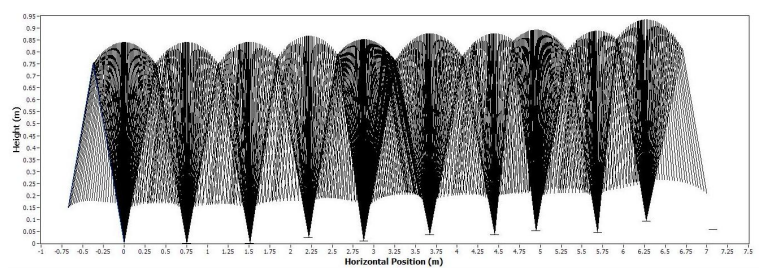

Fig. 10. Configuration, over time, of 10 irregularly spaced steps. The step length is a Gaussian random variable, $N(0.75,0.1)$ resulting here in an interval $[0.494,0.799] \mathrm{m}$ and step height is $N(0.0,0.025)$ resulting here in the interval $[-0.037,0.045] \mathrm{m}$. 


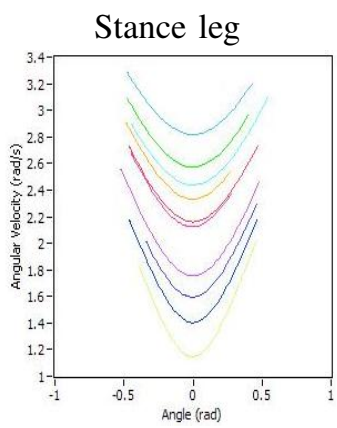

(a)

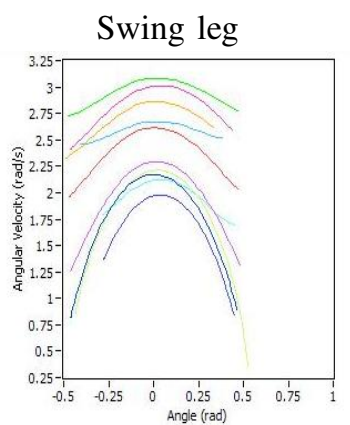

(b)
Fig. 11. Hybrid phase space trajectories for irregularly spaced footholds with variations in 2-dimensions.

\section{B. Relation to more complex robot architectures}

Complex multi-link walking robots (that we earlier referred to as anchor models) have been controlled in various ways, some more complex than others. In nature, these strategies seem to be acquired in an incremental fashion [15], so that multiple primitives may be reused [1] and multiple global objectives may be achieved in a straight-forward manner. The goal of our work has been to define a strategy that is constructively designed using primitives that are biologically plausible. We believe that the modular nature of the strategy and the associated understanding of the dynamics would ultimately enable implementation in more complex multi-link system (e.g., a humanoid robot with ankles, knees and hips) despite imprecision in available models and constraints on controller complexity - unavoidable problems that have been difficult to deal with in traditional approaches to robust nonlinear control. Demonstrating this claim is the focus of our current research work.

\section{CONCLUSIONS}

In this paper we present a novel approach to encoding the task of dynamic walking. This methodology, based on the use of natural dynamics, parsimonious active control and biological inspiration, provides a natural and efficient way to adapt to irregular terrain. At a higher level, we view this work as a step towards a general theory of control strategies for dynamically dexterous robots that behave robustly, efficiently and gracefully through the intelligent use of natural dynamics.

\section{ACKNOWLEDGMENT}

This work has taken place in the Intelligent Robotics Lab at the Artificial Intelligence Laboratory, The University of Texas at Austin. Research of the Intelligent Robotics lab is supported in part by grants from the National Science Foundation (IIS0413257 and IIS-0538927), from the National Institutes of Health (EY016089), and by an IBM Faculty Research Award.
[2] J. Pratt, C-M. Chew, A. Torres, P. Dilworth, and G. Pratt, "Virtual model control: An intuitive approach for bipedal locomotion". Int. J. Robotics Research, Vol. 20(2), pp.129-143, 2001.

[3] M. Raibert, Legged Robots that Balance, The MIT Press, Cambridge, MA, 1986.

[4] R. M. Ghigliazza, R. Altendorfer, P. Holmes and D. Koditschek, "A simply stabilized running model", SIAM Review, Vol. 47(3), pp. 519549, 2005.

[5] J.K. Hodgins and M. Raibert, "Biped gymnastics", Int. J. Robotics Research, Vol. 9(2), pp. 115-132, 1990.

[6] M. Garcia, A. Ruina, A. Chatterjee and M. Coleman, "The simplest walking model: stability, complexity, and scaling", ASME J. Biomechanical Engineering, Vol. 120(2), pp. 281-288, Apr 1998.

[7] J-J. Slotine, W. Li, Applied Nonlinear Control, Pearson Education, 1991.

[8] E. R. Westervelt, G. Buche, and J. W. Grizzle, "Experimental Validation of a Framework for the Design of Controllers that Induce Stable Walking in Planar Bipeds", The International Journal of Robotics Research, Vol. 24(6), pp. 559-582, 2004.

[9] S.H. Collins, A. Ruina, R. Tedrake and M. Wisse, "Efficient bipedal robots based on passive-dynamic walkers", Science, Vol. 307, pp. 10821085, Feb. 2005.

[10] D. Koditschek, "Task encoding: toward a scientific paradigm for robot planning and control", Robotics and Autonomous Systems, Vol. 9, pp. 5-39, 1992.

[11] T. McGeer, "Dynamics and control of bipedal locomotion", J. Theoretical Biology, Vol. 163, pp. 277-314, 1993.

[12] M. Coleman and A., Ruina, "An uncontrolled toy that can walk but cannot stand still", Physical Review Letters, Vol. 80(16), pp. 3658-3661, Apr 1998.

[13] M. Wisse, Three additions to passive dynamic walking; actuation, an upper body, and 3D stability, Proc. IEEE Intl. Conf. Humanoid Robots, 2004.

[14] R.J. Full and D.E. Koditschek, "Templates and anchors: neuromechanical hypotheses of legged locomotion on land", J. Experimental Biology, Vol. 202(23), pp. 3325-3332, 1999.

[15] Y.P. Ivanenko, N. Dominici, G. Cappellini, B. Dan, G. Cheron and F. Lacquaniti, "Development of pendulum mechanism and kinematic coordination from the first unsupported steps in toddlers", J. Experimental Biology, Vol. 207, pp. 3797-3810, 2004.

[16] G.A. Cavagna, P.A. Willems, M.A. Legramandi and N.C. Heglund, "Pendular energy transduction within the step in human walking", $J$. Experimental Biology, Vol. 205, pp. 3413-3422, 2002.

[17] R.M. Alexander, Principles of Animal Locomotion, Princeton University Press, 2002.

[18] B.J. Kuipers and S. Ramamoorthy, "Qualitative modeling and heterogeneous control of global system behavior", In C. J. Tomlin and M. R. Greenstreet (Eds.), Hybrid Systems: Computation and Control, Lecture Notes in Computer Science 2289, Springer Verlag. 2002.

[19] S. Ramamoorthy and B.J. Kuipers, "Qualitative heterogeneous control of higher order systems", In O. Maler and A. Pnueli (Eds.), Hybrid Systems: Computation and Control, Lecture Notes in Computer Science 2623, Springer Verlag. 2003.

[20] S. Mochon and T.A. McMahon, "Ballistic walking: An improved model", Mathematical Biosciences, Vol. 52, pp. 241-260, 1980.

[21] A.D. Kuo, J.M. Donelan and A. Ruina, "Energetic consequences of walking like an inverted pendulum: step-to-step transitions", Exercie and Sport Sciences Review, Vol. 33(2), pp.88-97, 2005.

[22] G.J. Sussman and J. Wisdom, Structure and Interpretation of Classical Mechanics, The MIT Press, 2001.

[23] A.D. Kuo, Energetics of actively powered locomotion using the simplest walking model, J. Biomechanical Engineering, Vol. 124, pp. 113-120, 2002.

[24] J.M. Winters and P.E. Crago (Eds.), Biomechanics and Neural Control of Posture and Movement, Springer-Verlag, New York, 2000.

[25] G.M. Zaslavsky, Hamiltonian Chaos and Fractional Dynamics. Oxford University Press, New York, 2005.

[26] J. Mawhin, M. Willem, Critical Point Theory and Hamiltonian Systems, Springer-Verlag, New York, 1989.

\section{REFERENCES}

[1] M.H. Dickinson, C.T. Farley, R.J. Full, M.A.R. Koehl, R. Kram and S. Lehman, "How animals move: An integrative view", Science, Vol. 288, pp. 100-106, April 2000. 